SHEP 97-03

\title{
Large $N$ and the Renormalization Group
}

\author{
Marco D'Attanasio and Tim R. Morris \\ Department of Physics, University of Southampton, \\ Highfield, Southampton SO17 1BJ, UK
}

\begin{abstract}
In the large $N$ limit, we show that the Local Potential Approximation to the flow equation for the Legendre effective action, is in effect no longer an approximation, but exact - in a sense, and under conditions, that we determine precisely. We explain why the same is not true for the Polchinski or Wilson flow equations and, by deriving an exact relation between the Polchinski and Legendre effective potentials (that holds for all $N$ ), we find the correct large $N$ limit of these flow equations. We also show that all forms (and all parts) of the renormalization group are exactly soluble in the large $N$ limit, choosing as an example, $D$ dimensional $O(N)$ invariant $N$-component scalar field theory. $\dagger$
\end{abstract}

hep-th/9704094

April, 1997.

$\dagger$ This footnote does not appear in the version to be published in Phys. Lett. B. 


\section{Introduction.}

The large $N$ limit 1 in combination with the exact Renormalization Group (RG) has the potential to be a very powerful non-perturbative technique. The exact solution of the large $N$ limit along these lines offers a tractable and possibly more general alternative to the traditional approach of introducing collective (bound state) fields [1]. Indeed, as we will show, the exact solution of the large $N$ limit of the $\mathrm{RG}$ flow equations does not require the introduction of collective fields, and yields solutions of a more general type than have been considered up until now.

We will show how to solve exactly for the functional dependence of the full effective action in the large $N$ limit. However, of particular interest is the flow equation for the potential, which closes in the large $N$ limit, under certain conditions, and is closely related to the Local Potential Approximation (LPA). A number of interesting results concerning the LPA and this connection, have already been derived in refs. [2]-[11], however, to our knowledge there is no derivation of the general conditions, over and above the large $N$ limit, under which sufficient simplifications occur to allow exact solution of the RG equations, and/or such that these simplifications coincide with the LPA. The main point of the present paper is to derive these conditions.

Specifically, we will show that the flow equations for $N$ component $O(N)$ invariant scalar field theory simplify if and only if the one-particle irreducible part of the interaction part of the effective action, a.k.a. $\Gamma_{\Lambda}$ (to be defined below), is a functional only of the square of the field $\varphi^{2}(\mathbf{x})=\varphi_{a}(\mathbf{x}) \varphi_{a}(\mathbf{x})$ at some point $\Lambda$. Under rather general conditions, as discussed later, this occurs if the interaction part of the bare action, $S_{\Lambda_{0}}$, is the (generalised) Legendre transform [12] of such a Legendre effective action, viz. $\Gamma_{\Lambda_{0}} \equiv \Gamma_{\Lambda_{0}}\left[\varphi^{2}\right]$. This space of bare actions is indeed much larger than the one considered in traditional large $N$ methods [1], and in derivations of the large $N$ flow equations that start from these methods [3] [5] [7] [10], where the bare interactions were restricted to a general bare potential.

Although we concentrate on $O(N)$ scalar field theory, it will be clear that the derivations (and proofs) easily generalise to other field theories.

It is worth stressing that the large $N$ limit of the Legendre effective action does not reduce simply to the sum of a potential and a standard kinetic term. On the contrary, the Legendre effective action generally contains contributions to all orders in derivatives even in this large $N$ limit (as we will see later2). Since the approximation in the LPA [15] [16] [17]

$1 N$ being the number of fields in a quantum field theory

${ }^{2} O\left(\partial^{2}\right)$ results for general $N$, including $N=\infty$ [13], were announced in ref. [14]. 
is the neglect of all these higher order terms, the LPA in the large $N$ limit is even in this case strictly speaking incorrect. However, the proof of exact reduction of the Legendre flow equation to that for just an effective potential in the large $N$ limit, arises by considering only constant fields (i.e. ones that do not vary in space-time) and as we will see, so far the reduced equations coincide with the equations obtained in the LPA.

We will show that the correct large $N$ limit of the Wilson flow equations 18], or equivalently[19] the large $N$ limit of Polchinski's flow equations [20] for the effective potential, does not at all coincide with the LPA. We will show that the flow equations for the potential do again close in the large $N$ limit however, and we derive the correct equations through a generalised Legendre transform relationship between the Polchinski effective potential and the Legendre effective potential, which we formulate here. The generalised Legendre transform relationship, first written down by one of us in ref.[12], is the crucial step in this derivation. The fact that the LPA is not the correct large $N$ limit in this case is remarkable, given that the LPA of the Polchinski equation nevertheless provides the right eigenvalue spectrum for the Wilson-Fisher fixed point and the correct behaviour for the BMB[21] phenomenon [11]. However on the one hand, we list several instances in which the LPA of the Polchinski equation gives incorrect results, and on the other hand, we show that flow equations of a very general form (of which the LPA is one instance and the correct equation another) all yield the correct results for the eigenvalue spectrum of the Gaussian fixed point, the line of Gaussian fixed points that appear at $N=\infty$ in upper critical dimensions less than four [11] 21], and the Wilson-Fisher fixed point. In so doing, we also show that all the present forms of the RG can be straightforwardly solved for the corresponding RG eigenvalues.

We finish this introduction with a brief discussion of earlier work on the large $N$ limit of the RG[2]- [11], in order to further clarify its relation to the present paper. In the classic paper of Wegner and Houghton[2], it was already recognized that the effective potential for the Wilson-Fisher fixed point, and its perturbations, were exactly soluble. They noted that the functional flow equation could be exactly reduced to a partial differential equation for the effective potential. Later it was noticed that all points on the flow were soluble, for any bare potential in this so-called LPA [4] (see also refs. [5] [6] [11]). Actually, the exact solution was already published and analysed by Ma[3], who solved for any finite renormalization group step for the effective potential by now-standard large $N$ methods. These exact results were all derived for the potential only and for sharp cutoff. Apart from a solution 
for a particular smooth cutoff 13], we know of no other published exact results3 extracted from the large $N$ flow equations. As we have already stated, we will show in fact that it is possible to go well beyond this: that all the equations are soluble for any cutoff, both around the Wilson-Fisher fixed point and general points, and indeed not just for the potential but for the full effective action.

In ref. [10, the expansion of the potential about its minimum was investigated and shown to yield a partial system of 'perfect coordinates' for the RG. This property of perfect coordinates leads to an elegant derivation of the RG eigenvalues, which we will utilise later, for we will show that this property holds not just for sharp cutoff (viz. the Wegner-Houghton RG[2]) but for all cutoffs and all present forms of the RG. By thus showing that truncations of the Taylor expanded flow equations around the minimum of the potential, become exact in the limit $N \rightarrow \infty$, we also gain some insight into why truncations around the minimum give good results in finite $N$ scalar field theory [8] [22].

\section{The large $N$ limit of the Legendre flow equation.}

As stated earlier, for concreteness we will restrict our attention to an $N$-component $O(N)$ invariant scalar field theory. However, the appropriate generalizations to the other classical groups, and other (matter) fields is straightforward.

Consider the partition function, with kinetic term modified to include an infrared cutoff:

$$
\exp \mathcal{W}[J]=\int \mathcal{D} \varphi \exp \left\{-\frac{1}{2} \varphi_{a} \cdot \Delta_{I R}^{-1} \cdot \varphi_{a}-S_{\Lambda_{0}}[\varphi]+J_{a} \cdot \varphi_{a}\right\}
$$

We have introduced $\Delta_{I R}(q, \Lambda)=C_{I R}\left(q^{2} / \Lambda^{2}\right) / q^{2}$, where $C_{I R}$ is the infrared cutoff function. The subscript ${ }_{a}$ is the internal index running from 1 to $N$, and $S_{\Lambda_{0}}$ stands for the $O(N)$ invariant bare interactions. Some more or less pedantic statements, on notation, and $C_{I R}$, are given in appendix A. Differentiating (1) with respect to $\Lambda$ and rewriting in terms of $\Gamma_{\Lambda}$, the interaction part of the Legendre effective action, gives [12] [19] [23]:

$$
\begin{aligned}
\frac{\partial}{\partial \Lambda} \Gamma_{\Lambda}[\varphi] & =-\frac{1}{2} \operatorname{tr} \frac{1}{\Delta_{I R}} \frac{\partial \Delta_{I R}}{\partial \Lambda} \cdot\left(A^{-1}\right)_{a a} \\
\text { where } \quad A_{a b} & =\delta_{a b}+\Delta_{I R} \cdot \frac{\delta^{2} \Gamma_{\Lambda}}{\delta \varphi_{a} \delta \varphi_{b}}
\end{aligned}
$$

3 In ref. [8] the large $N$ equation was further approximated and solved for. The authors speculate that this approximation may become exact for a certain choice of cutoff function. However, this is not correct since the approximated integral $L_{1}^{3}\left(u^{\prime}\right)$ [8] has a cut from $u^{\prime}=-1$ for all sensible cutoffs, not a double pole at $u^{\prime}=-1$ as the authors claimed. 
Here $\Gamma_{\Lambda}$ is defined by $\Gamma_{\Lambda}[\varphi]+\frac{1}{2} \varphi_{a} \cdot \Delta_{I R}^{-1} \cdot \varphi_{a}=-\mathcal{W}[J]+J_{a} \cdot \varphi_{a}$, where now $\varphi_{a}=\delta \mathcal{W} / \delta J_{a}$ is the classical field.

Suppose that the interaction part of the Legendre effective action is just a functional of $\varphi_{a}(\mathbf{x}) \varphi_{a}(\mathbf{x})$, i.e. $\Gamma_{\Lambda}=\Gamma_{\Lambda}\left[\varphi^{2}\right]$ (at least at some value of $\Lambda$ ), then we have

$$
\frac{\delta^{2} \Gamma_{\Lambda}}{\delta \varphi_{a}(\mathbf{x}) \delta \varphi_{b}(\mathbf{y})}=2 \delta_{a b} \delta(\mathbf{x}-\mathbf{y}) \frac{\delta \Gamma_{\Lambda}}{\delta \varphi^{2}(\mathbf{y})}+4 \varphi_{a}(\mathbf{x}) \varphi_{b}(\mathbf{y}) \frac{\delta^{2} \Gamma_{\Lambda}}{\delta \varphi^{2}(\mathbf{x}) \delta \varphi^{2}(\mathbf{y})}
$$

We note that this is of the form $a \delta_{a b}+b_{a b}$. If $\Gamma_{\Lambda}$ has a smooth limit as $N \rightarrow \infty$, then it is easy to see that the second term $b_{a b}$ contributes negligably in this limit. Indeed, whereas the other terms in (3i) and (2b) yield a factor of $\delta_{a a}=N$ on taking the inverse and tracing in (2a), corrections resulting from $b_{a b}$ have no $\delta_{a a}$ component to all orders in $b$ : $\left(a \delta_{a b}+b_{a b}\right)^{-1}=a^{-1} \delta_{a b}-a^{-1} b_{a b} a^{-1}+a^{-1} b_{a c} a^{-1} b_{c b} a^{-1}-\cdots$. Therefore to leading order in $N$, equation (2) simplies to

$$
\frac{\partial}{\partial \Lambda} \Gamma_{\Lambda}[\varphi]=-\frac{N}{2} \operatorname{tr} \frac{1}{\Delta_{I R}} \frac{\partial \Delta_{I R}}{\partial \Lambda} \cdot A^{-1}
$$

where now

$$
A(\mathbf{x}, \mathbf{y})=\delta(\mathbf{x}-\mathbf{y})+2 \Delta_{I R}(\mathbf{x}-\mathbf{y}) \frac{\delta \Gamma_{\Lambda}}{\delta \varphi^{2}(\mathbf{y})}
$$

(the propagator $\Delta_{I R}$ having been Fourier transformed into position space). Two points are now obvious from (4). Firstly, if for some value $\Lambda, \Gamma_{\Lambda}$ is a functional only of $\varphi^{2}$, then since (4b) is also only a functional of $\varphi^{2}$, and (4a) a first order differential equation in $\Lambda$, we have immediately that this form of $\Gamma_{\Lambda}$ is preserved by the flow, i.e. $\Gamma_{\Lambda} \equiv \Gamma_{\Lambda}\left[\varphi^{2}\right]$ for all $\Lambda$. Secondly, in order to obtain a finite non-trivial limit as $N \rightarrow \infty$, we must transform

$$
\Gamma_{\Lambda} \mapsto N \Gamma_{\Lambda}, \quad \text { and consequently } \quad \varphi_{a} \mapsto \sqrt{N} \varphi_{a}
$$

Of course these are nothing but the standard transformations [1], but we have here yet another derivation of them. The net effect of this is to remove the factor of $N$ from (4a). We assume from now on that this has been done.

The fact that the flow equation (4) now contains only zero or one point functions allows for a considerable simplification: Set $\varphi^{2}(\mathbf{x})=z$, where $z$ is a constant. Regarding the effective action as given by a derivative expansion (or otherwise), we see that only the potential term of $\Gamma_{\Lambda}=\int d^{D} x V\left(\varphi^{2}, \Lambda\right)+\cdots$ survives in (4), which thus takes the form

$$
\frac{\partial}{\partial \Lambda} V(z, \Lambda)=-\frac{\Omega}{2} \int_{0}^{\infty} d q q^{D-1} \frac{1}{\Delta_{I R}} \frac{\partial \Delta_{I R}}{\partial \Lambda} \frac{1}{1+2 \Delta_{I R} V^{\prime}(z, \Lambda)}
$$


(where in here $\Delta_{I R} \equiv \Delta_{I R}(q, \Lambda)$, prime refers to differentiation with respect to $z$, and $\Omega(2 \pi)^{D}$ is the solid angle of a $(D-1)$-sphere).

Evidently (6) coincides with what would be obtained in the Local Potential Approximation, since this results from the ansatz $\Gamma_{\Lambda}=\int d^{D} x V\left(\varphi^{2}, \Lambda\right)$, combined with the requirement that all higher derivative terms are discarded. Nevertheless contributions from all higher derivatives are most certainly (generally) there, as is evident from the full equation (4).

The conditions under which this 'reduction to LPA' takes place, follow from the first point made below (4), i.e. a sufficient condition is that $\Gamma_{\Lambda}=\Gamma_{\Lambda}\left[\varphi^{2}\right]$ at some point $\Lambda$. In particular, if the ultraviolet cutoff is absorbed in $C_{I R}$, by modifying it so that e.g. $C_{I R}(q, \Lambda)=0$ for all $q>\Lambda_{0}$ then we have that $\Gamma_{\Lambda_{0}}=S_{\Lambda_{0}}$ [12], and thus the reduction occurs if the bare interactions are functionals only of $\varphi^{2}$. (If the ultraviolet cutoff is not so absorbed in $C_{I R}$, then generally $\Gamma_{\Lambda} \rightarrow S_{\Lambda_{0}}$ as $\Lambda \rightarrow \infty$ and the conclusions will be the same.) One can convince oneself that the condition $\Gamma_{\Lambda}=\Gamma_{\Lambda}\left[\varphi^{2}\right]$ at some point $\Lambda$, is also necessary: If $\Gamma_{\Lambda}$ contains a term of the form

$$
\int d^{D} x f\left(\varphi^{2}\right) \varphi_{a} B \varphi_{a}
$$

where $f$ is some function and $B \equiv B(-i \partial)$ a two-point function satisfying $B(0)=0$, here expressed as a derivative operator, then (3) receives corrections containing $\delta_{a b}$. These corrections thus survive to (田), and furthermore, the $\delta_{a b}$ term arising from differentiating the two fields in $\varphi_{a} B \varphi_{a}$, modifies (6) in way which can only be quantified once $B$ and $f$ are known. It is only contributions to the potential, and terms (or parts of terms) that can be put in form (7) that survive the twin reductions of the large $N$ limit and setting the field to a constant. This fact however does not appear to allow any widening of the above condition, such that the equations remain soluble, because terms of the form (17) receive their corrections through the flow equation from many other forms of terms and no closure appears possible in general. In particular therefore, it appears that no closure allows the computation of a non-trivial $f$ and $B$ in general, nor does a closure exist which guarantees that these terms vanish, beyond the already stated sufficient condition.

\section{The LPA of Polchinski's flow equation.}

Consider now Polchinski's flow equation for the interaction part $S_{\Lambda}[\Phi]$ of the Wilsonian effective action $S_{\Lambda}^{e f f}=\frac{1}{2} \Phi_{a} \cdot \Delta_{U V}^{-1} \cdot \Phi_{a}+S_{\Lambda}$. This is given by [20] [12]

$$
\frac{\partial S_{\Lambda}}{\partial \Lambda}=\frac{1}{2} \frac{\delta S_{\Lambda}}{\delta \Phi_{a}} \cdot \frac{\partial \Delta_{U V}}{\partial \Lambda} \cdot \frac{\delta S_{\Lambda}}{\delta \Phi_{a}}-\frac{1}{2} \operatorname{tr} \frac{\partial \Delta_{U V}}{\partial \Lambda} \cdot \frac{\delta^{2} S_{\Lambda}}{\delta \Phi_{a} \delta \Phi_{a}}
$$


$\Delta_{U V}(q, \Lambda)=C_{U V}(q, \Lambda) / q^{2}$, and $C_{U V}$ is an effective ultra-violet cutoff function. Its properties (and that of $\Phi$ ) are recalled in appendix A. We remind the reader that Wilson's flow equation [18] is identical to (8), after the transformation $\Phi_{a} \mapsto \sqrt{C_{U V}} \Phi_{a}$ (and $\mathcal{H} \equiv-S_{\Lambda}$ ) [19] [14, and therefore does not need separate discussion. Similarly, the Wegner-Houghton equation arises as the sharp cutoff limit of eqn.(8) [12] [14].

A finite large $N$ limit is achieved with the analagous transformations to (5), viz.

$$
S_{\Lambda} \mapsto N S_{\Lambda} \quad, \quad \Phi_{a} \mapsto \sqrt{N} \Phi_{a}
$$

[Again these either follow from standard methods, or may be deduced directly, this time by requiring the order $N$ parts of the quantum (trace) term to balance the classical $\left(\sim S_{\Lambda}^{2}\right)$ term and the left hand side in eqn.(8).] Suppose once more that we assume that the interactions are restricted to the form $S_{\Lambda}=S_{\Lambda}\left[\Phi^{2}\right]$, at some value of $\Lambda$. Combining this with (9), and $N \rightarrow \infty$, we obtain

$$
\begin{aligned}
\frac{\partial}{\partial \Lambda} S_{\Lambda}[\Phi] & =\int d^{D} x\left\{2\left(\frac{\delta S_{\Lambda}}{\delta \Phi^{2}} \Phi_{a}\right) \frac{\partial \Delta_{U V}}{\partial \Lambda}(-i \partial, \Lambda)\left(\Phi_{a} \frac{\delta S_{\Lambda}}{\delta \Phi^{2}}\right)-\gamma \Lambda^{D-3} \frac{\delta S_{\Lambda}}{\delta \Phi^{2}}\right\} \\
\text { where } \quad \gamma & =\Omega \Lambda^{3-D} \int_{0}^{\infty} d q q^{D-1} \frac{\partial \Delta_{U V}}{\partial \Lambda}(q, \Lambda)
\end{aligned}
$$

Because in this limit, the only field derivatives are first order, we have again a reduction to LPA on setting $\Phi_{a}(\mathbf{x})$ constant, $\Phi^{2}(\mathbf{x})=y$ (not to be confused with the coordinate $\mathbf{y}[!]$ ):

$$
\begin{aligned}
\frac{\partial}{\partial \Lambda} U(y, \Lambda) & =\frac{4 \alpha}{\Lambda^{3}} y\left(U^{\prime}\right)^{2}-\gamma \Lambda^{D-3} U^{\prime} \\
\text { where } \quad \alpha & =-C_{U V}^{\prime}(0)
\end{aligned}
$$

(the prime on $C_{U V}$ meaning differentiation with respect to its argument). Nevertheless, eqn.(11) is not correct (for all $\Lambda$ ). This is because the form $S_{\Lambda}=S_{\Lambda}\left[\Phi^{2}\right]$ is violated by the first term on the right hand side of (10a) and is thus not preserved by the flow. Independently, there are several ways to see that (11) cannot be the exact equation. Firstly, it fails to give the correct $\beta$ function in $D=4$ dimensions [14] [24]. Secondly, there is nothing wrong with a cutoff function satisfying $C_{U V}^{\prime}(0)=0$, but (11) collapses to a linear equation which cannot be right since in particular it cannot support non-trivial fixed points. the sharp cutoff limit results in $\alpha=0$, where the exact equation should collapse to the LPA of Wegner-Houghton equation [15] [16][17]. Finally, a general Legendre transform

4 In fact it is straightforward to solve the $\alpha=0$ case and confirm its trivial behaviour. 
relationship exists between the Polchinski and Legendre effective actions [12] [17] [25] which implies that precisely when $\alpha=0$, the Polchinski and Legendre effective potentials should be identical.

\section{Legendre transform relations.}

Here, we develop the consequences of the Legendre transform relationship for the two effective potentials. The content of this section holds generally, viz. not just for large $N$, with the exception of the scaling form for the cutoffs which, for smooth cutoffs, is only appropriate for fixed points and fields with no anomalous scaling (c.f. refs. 19 17] 14 and appendix A) - the generalization is straightforward however.

The Polchinski (or Wilson) effective action has a tree structure 20] [12] whose oneparticle irreducible parts are given by the infrared cutoff Legendre effective action introduced previously [12], providing that we set $C_{I R}\left(q^{2} / \Lambda^{2}\right)=1-C_{U V}\left(q^{2} / \Lambda^{2}\right)$. The interactions are then related by the Legendre transform relationship 12

$$
S_{\Lambda}[\Phi]=\Gamma_{\Lambda}[\varphi]+\frac{1}{2}\left(\varphi_{a}-\Phi_{a}\right) \cdot \Delta_{I R}^{-1} \cdot\left(\varphi_{a}-\Phi_{a}\right)
$$

which by differentiation, implies the following functional relations between the two fields:

$$
\begin{aligned}
\varphi_{a} & =\Phi_{a}-\Delta_{I R} \cdot \frac{\delta S_{\Lambda}}{\delta \Phi_{a}} \\
\text { and } \quad \Phi_{a} & =\varphi_{a}+\Delta_{I R} \cdot \frac{\delta \Gamma_{\Lambda}}{\delta \varphi_{a}} .
\end{aligned}
$$

It is straightforward to show that the flow equations are transformed into each other by (13); By differentiating (14a) with respect to $\Phi$, and (14b) with respect to $\varphi$, one derives

$$
\left(A^{-1}\right)_{a b}=\delta_{a b}-\Delta_{I R} \cdot \frac{\delta^{2} S_{\Lambda}}{\delta \Phi_{a} \delta \Phi_{b}}
$$

then e.g. differentiating (13) with respect to $\Lambda$ at constant $\Phi$, and using (14a) and the above relation, one derives (8) from (2) (up to an unimportant vacuum energy [20] [12]).

In the case of constant fields with $\Phi_{a}(\mathbf{x})=\hat{\Phi}_{a} \sqrt{y}$ and $\varphi(\mathbf{x})=\hat{\varphi}_{a} \sqrt{z}$ (where we have introduced the unit vectors $\left.\hat{\Phi}^{2}=\hat{\varphi}^{2}=1\right)$, (13) collapses to

$$
U(y, \Lambda)=V(z, \Lambda)+\frac{\Lambda^{2}}{2 \alpha}(\varphi-\Phi)^{2}
$$

and either directly from here or from (14), we see that $\hat{\varphi}_{a}=\hat{\Phi}_{a}$,

$$
\sqrt{\frac{y}{z}}=1+\frac{2 \alpha}{\Lambda^{2}} V^{\prime}(z, \Lambda) \quad \text { and } \quad \sqrt{\frac{z}{y}}=1-\frac{2 \alpha}{\Lambda^{2}} U^{\prime}(y, \Lambda)
$$


Combining the above equations gives,

$$
V^{\prime}(z, \Lambda)=\frac{U^{\prime}(y, \Lambda)}{1-2 \alpha U^{\prime}(y, \Lambda) / \Lambda^{2}} .
$$

We have in particular at $\alpha / \Lambda^{2} \equiv \Delta_{I R}(0)=0$, corresponding to an efficient infrared cutoff, that the constant fields and the effective potentials are equal: $y=z, U=V$ [12] [17]. Consequently, the Polchinski effective potential tends to the full 5 Legendre effective potential in the limit as $\Lambda \rightarrow 0$ [25]. For $\alpha>0$, (17) is compatible with the observed large field behaviour of the respective potentials: we have $V^{\prime} \sim \tilde{A} z^{2 /(D-2)}$, where $\tilde{A}$ is a dimensionless constant (by dimensions on assuming $\Lambda$ drops out of this limit) [19][25], and thus from (17), $U^{\prime} \sim \frac{\Lambda^{2}}{2 \alpha}-\frac{\Lambda^{4}}{4 \alpha^{2} A} z^{2 /(2-D)}$, concurring with derivative expansion estimates [26].

\section{The large $N$ limit of Polchinski's flow equation.}

Returning to (10a), we note that the form $S_{\Lambda} \equiv S_{\Lambda}\left[\Phi^{2}\right]$ is not preserved in the large $N$ limit, precisely because the one-particle reducible parts do not respect this form. The best one can derive for the full $S_{\Lambda}$, is its tree expansion in terms of one-particle irreducible vertices, the latter simplifying as a consequence of $\Gamma_{\Lambda} \equiv \Gamma_{\Lambda}\left[\varphi^{2}\right]$, e.g. we derive from (15) and (4b), at $\varphi=\Phi=0$ the large $N$ form for the effective two-point function.

However, for the large $N$ Polchinski effective potential we can, by using the results of the last section, derive an exact closed equation - either directly from (16), (17) and (6), along the lines outlined below (6), or by setting $\Phi_{a}=\hat{\varphi}_{a} \sqrt{y}$, in (8) and using (15), (46) and (17). Either way, we obtain

$$
\frac{\partial U}{\partial \Lambda}=\frac{4 \alpha}{\Lambda^{3}} y\left(U^{\prime}\right)^{2}+\frac{\Omega}{2}\left(\frac{2 \alpha}{\Lambda^{2}} U^{\prime}-1\right) \int_{0}^{\infty} d q q^{D-1} \frac{1}{\Delta_{I R}} \frac{\partial \Delta_{I R}}{\partial \Lambda} \frac{1}{1+2\left(\Delta_{I R}-\alpha / \Lambda^{2}\right) U^{\prime}} .
$$

\section{Exact large $N$ solutions.}

Exact large $N$ solutions of RG effective potentials have been found for: the sharp cutoff (Wegner-Houghton) case, around the Wilson-Fisher fixed point [2] [10] and at general points [5] [6] [11], the Legendre flow equation with a particular smooth cutoff - around the Wilson-Fisher fixed point [13], and the large $N$ LPA (11) for Polchinski's flow equation at general points[11]. In this section, we show that these equations, and (18), are exactly soluble for any cutoff, both around the Wilson-Fisher fixed point and at general points, and that this is possible not just for the potential, but also for the full effective action

5 i.e. without infrared cutoff 
$\Gamma_{\Lambda}\left[\varphi^{2}\right]$. At the same time, we will shed light on why (11) is capable of producing the correct exact results [11] despite the fact that (11) is not itself exact.

Since corrections are functionals of $\varphi^{2}$ only, the kinetic term $\sim\left(\partial_{\mu} \varphi\right)^{2}$ remains normalised, and the fields have no anomalous dimension. Thus we write $z \mapsto z \Lambda^{D-2}$, $V(z, \Lambda) \mapsto V(z, t) \Lambda^{D}, t=\ln (\mu / \Lambda)$ (where $\mu$ is an arbitrary finite energy scale), and similarly for $y$ and $U$, after which all powers of $\Lambda$ may be eliminated from (66), (11) and (18). Shifting to a stationary point $x_{0}(t)$, by writing $z=x_{0}(t)+x\left[y=x_{0}(t)+x\right]$, we define $W(x, t)=V^{\prime}\left[W(x, t)=U^{\prime}\right]$, with thus $W(0, t) \equiv 0$. Differentiating (66), (11), and (18) by $x$, all three flow equations can then be written (for general cutoff) in the form

$$
\dot{W}-\dot{x}_{0} W^{\prime}+(D-2)\left(x_{0}+x\right) W^{\prime}-2 W=\gamma W^{\prime}-\left(d\left[x+x_{0}\right]+c-\frac{d \gamma}{D-2}\right) W W^{\prime}+F \quad,
$$

where the dot 'stands for $\partial / \partial t$ (at constant $x$ ), and $F\left(W, W^{\prime}, x, t\right)$ is an analytic function of $W$ (around $W=0$ ), $W^{\prime}$ and $x$, of $O\left(W^{2}\right) . \gamma, d$ and $c$ (chosen in the above form for convenience), are numerical constants.

We now prove that all flow equations of form (19), yield the correct structure of RG fixed points in the sense that: There exists a Gaussian fixed point $W=0$, with RG eigenvalues $\lambda_{n}^{G}=2-(D-2) n$, which becomes a line of Gaussian fixed points in the upper critical dimensions $D_{k}=2+2 / k, k=2,3, \cdots$ [21] [11]. There exists a non-trivial (Wilson-Fisher - or Heisenberg) fixed point with eigenvalue spectrum $\lambda_{n}^{W F}=D-2(n+1), n=0,1, \cdots$ [2], - providing only that $c \neq 0$. Furthermore, if $c$ (for $D>2$ ) and $\gamma$ are positive, which we will assume, then the Wilson-Fisher fixed point derived from (19) exists, as expected, only in dimensions $2<D<4$. For our three flow equations, $\gamma=2 \Omega \int d q q^{D-1} C_{I R}^{\prime}\left(q^{2}\right)$, as in (106). $d=8 \alpha$ for the Polchinski types (11), (18), while $d=0$ for (6). $c=c_{L} \equiv$ $8 \Omega \int d q q^{D-3} C_{I R}^{\prime} C_{I R}$ for (6), $c=c_{P} \equiv 8 \alpha \gamma /(D-2)$ for (11), and $c=c_{L}+c_{P}-4 \alpha \gamma$ for (18). We note that for $C_{I R}$ monotonic, $\gamma, c_{L}$ and (for $D>2$ ) $c_{P}$ are guaranteed positive (c.f. appendix A), and (18)'s $c$ is positive at least in the range $2<D<4$.

Requiring $W(0, t)=0$ in (19), implies $W^{\prime}(0, t)=0$ and thus $W(x, t)=0$, unless

$$
\dot{x}_{0}=(D-2) x_{0}-\gamma
$$

$6 \quad$ N.B. By this we restrict ourselves to potentials with such stationary points, ruling out in particular the High Temperature fixed point solution[11] to $(8): U(y, t)=y /(2 \alpha)$.

7 by expanding in powers of $x$ or otherwise 
Substituting this back into (19), and expanding $W(x, t)=\sum_{n=1} W_{n}(t) x^{n}$, we obtain

$$
\begin{aligned}
\dot{W}_{1}+(D-4) W_{1} & =-\left[d x_{0}+c-\frac{d \gamma}{D-2}\right] W_{1}^{2}, \\
\dot{W}_{n}+[(D-2) n-2] W_{n} & =-\left[d x_{0}+c-\frac{d \gamma}{D-2}\right](n+1) W_{n} W_{1}+\cdots,
\end{aligned}
$$

where the second equation holds for $n>1$ and the dots stand for terms containing products of $W_{m}$ 's, $m<n$. Thus the $W_{n}(t)$ are all soluble in terms of lower $m W_{m}(t)$ and $x_{0}(t)$, and truncations to some finite $n$ are exact. This extends ref.[10]'s observation of 'perfect coordinates', to all cutoffs and flow equations. Consider now a fixed point: $W(x, t)=$ $W(x), x_{0}(t)=x_{0}$. From (20), $x_{0}=\gamma /(D-2)$ which, since $x_{0}$ is the original field squared, requires $D>2$. Substituting into (21), we find that either $W_{1}=0$ - which is discussed further below, or $W_{1}=(4-D) / c$ - which is the solution we take here. Since this implies that $W(x)$ crosses the $x$-axis only once, we must have $W_{1}>0$, and thus $D<4$, so that $W(x)>0$ for $x>0$. Otherwise, from the large field behaviours below (17), the potential (if it exists) ${ }^{8}$ is unbounded below. Substituting these solutions in (22), we see that the $W_{n}$ exist and are unique, with no further conditions. Now, from (20), we see that about this fixed point, there is an eigen-perturbation with $\delta x_{0}(t) \propto \mathrm{e}^{\lambda_{0}^{W F} t}$. From (21) and (22), there is just one new eigen-perturbation for each new $n=1,2, \cdots$, and this takes the form $\delta x_{0}(t)=0, \delta W_{m}(t)=0 \forall m<n$, and $\delta W_{n}(t) \propto \mathrm{e}^{\lambda_{n}^{W F} t}$. This completes the space of perturbations, and the eigenvalue spectrum of the Wilson-Fisher fixed point.

Now we return to the fixed point solution $W_{1}=0$. It is easy to see (by induction in increasing $n$ ) that at generic dimension $D$, (22) implies $W_{n}=0 \forall n$, i.e. the Gaussian fixed point. Since $W_{1}=0$, the eigenvalues of the perturbations are just given by the coefficient on the left hand sides of (21) and (22): $\lambda_{n}=\lambda_{n}^{G}$. But at $D=D_{k}$, the coefficient of $W_{k}$ for $n=k$ in (22), vanishes. Therefore in this case $W_{k}$ can be arbitrary. Since $W_{1}=0$, the eigenspectrum remains that of the Gaussian fixed point; $W_{k}$ is an exactly marginal coupling and the fixed point has become a line. (N.B. Since the coefficient of the $x$ terms in (19) vanish as a consequence of $W_{1}=0$, (20) does not yield an eigenperturbation. The mass perturbation, $\delta W(x, t) \propto \mathrm{e}^{\lambda_{0}^{G} t}$, on the other hand, exists but was excluded by our condition $W(0, t)=0$.)

Note that these proofs do not provide closed forms for non-trivial $W(x)$, or establish that they are non-singular for all $x+x_{0}>0$ (and thus in particular the extent of the

8 In fact such potentials typically encounter a singularity at some point 14 27] 11]. 
line of Gaussian fixed points at $D=D_{k}$ ) nor similarly, that the perturbations $\delta W(x, t)$ are acceptable [14] [27]. This can be established from general properties of the equations, similarly to the proofs in refs. [2] 28], or by deriving exact implicit solutions. Indeed these are easily achieved for all initial conditions and all $\Lambda$ : For simplicity restricting to the Legendre flow equation and before scaling, ${ }^{6}$ we differentiate (6) with respect to $z$ and write $W(z, \Lambda)=V^{\prime}(z, \Lambda)$, after which (6) takes the form $\partial W / \partial \Lambda=\mathcal{A}(W, \Lambda) W^{\prime}$. Now by change of dependent variables (or the method of characteristics), the general solution is

$$
z=\mathcal{F}(W(z, \Lambda))-\int^{\Lambda} d \chi \mathcal{A}(W(z, \Lambda), \chi)
$$

where $\mathcal{F}$ is an arbitrary function. A closely related technique works for the full effective action $\Gamma_{\Lambda}\left[\varphi^{2}\right]$. Let us define a Legendre transform with respect to $\varphi^{2}(\mathbf{x})$, introducing a source $K(\mathbf{x})$. Writing $\mathcal{W}[K]=-\Gamma_{\Lambda}\left[\varphi^{2}\right]+K \cdot \varphi^{2}$, we derive in standard fashion $\frac{\delta \Gamma_{\Lambda}}{\delta \varphi^{2}}=K$, and thus using (四) we obtain an equation for $\left.\frac{\partial \mathcal{W}}{\partial \Lambda}\right|_{K}$ whose general solution is immediate:

$$
\mathcal{W}=\mathcal{W}_{0}[K]+\frac{1}{2} \int^{\Lambda} d \chi \operatorname{tr} \frac{1}{\Delta_{I R}} \frac{\partial \Delta_{I R}}{\partial \chi} \cdot A^{-1},
$$

where $\mathcal{W}_{0}$ is an arbitrary $\Lambda$ independent functional of $K, \Delta_{I R} \equiv \Delta_{I R}(q, \chi)$ and from (4b), $A(\mathbf{x}, \mathbf{y})=\delta(\mathbf{x}-\mathbf{y})+2 \Delta_{I R}(\mathbf{x}-\mathbf{y}) K(\mathbf{y})$.

We finish with two remarks. Firstly, it looks quite plausible that the 'perfect coordinate' method (20)-(22) can be generalised to the full functional equation (44). Secondly, we are aware that a number of the results we have given may be interpreted and even derived at the diagrammatic level, however the derivations we give are compact, and non-perturbative, and are thus more powerful.

\section{Acknowledgements}

TRM acknowledges support of the SERC/PPARC through an Advanced Fellowship, and PPARC grant GR/K55738.

9 It is straightforward to generalise, - or use (17) if necessary, and scale $\Lambda$ out afterwards. 


\section{Appendix A. Notation and cutoffs.}

We use a condensed notation wherever convenient so two-point functions are often regarded as matrices in position or momentum (q) space, one-point functions as vectors, and contractions indicated by a dot. We work in $D$ dimensions. The partition function is assumed to be regulated by an overall cutoff $\Lambda_{0}$, but all equations of interest will be regulated by the intermediate cutoff $\Lambda$, and are thus completely insensitive to $\Lambda_{0}$ in the continuum limit $\Lambda_{0} \rightarrow \infty$. For more details see refs. [12] [19] [16] [17].

A more general propagator than $1 / q^{2}$ could be used (as multiplier to $C_{I R}$ and $C_{U V}$ ) without any significant changes, but at the price of some loss of clarity. Similarly the cutoff functions could be assumed to be general functions $C(q, \Lambda)$ of yet to be determined dimension, but since in the large $N$ limit for scalar fields no anomalous dimension remains, and since we will later in the paper be interested in (scale-free) critical points we simplify from the start to $C \equiv C\left(q^{2} / \Lambda^{2}\right)$.

The infrared cutoff function $C_{I R}$ satisfies $C_{I R}(r) \rightarrow 1$ as $r \equiv q^{2} / \Lambda^{2} \rightarrow \infty$, so that physics is independent of the infrared cutoff at scales much larger than $\Lambda$. In fact we require also that $C_{I R}(r) \rightarrow 1$ sufficiently fast that the momentum integrals in the effective action flow equations (2) are well regulated. For $r \rightarrow 0$, we require $C_{I R}(r) \rightarrow 0$, so that the infrared physics is indeed cutoff. In particular we will require that this happens fast enough to guarantee that a momentum Taylor expansion (a.k.a. derivative expansion) exists for the effective action (i.e. that there are no remaining massless modes). By unitarity, the cutoff functions must be analytic at $r=0$, (so that they hide no other massless modes).

The effective ultraviolet cutoff function $C_{U V}(r)$ is required to satisfy $C_{U V} \rightarrow 1$ as $r \rightarrow 0$, so that physics is unchanged at scales much less than the effective cutoff $\Lambda$, while for $r \rightarrow \infty, C_{U V}$ is required to vanish sufficiently fast that all momentum integrals are well regulated. In fact, we will insist that $C_{U V}(r)+C_{I R}(r)=1$ for all $r$, since then an intimate relation exists [12] between the Polchinski and Legendre flow equations. The field $\Phi=\Delta_{I R} \cdot J+\varphi$ is not the same as the field $\varphi$ in (1) [12], unless Polchinski's conditions 20] are applied to $C_{U V}$ and $J$. These conditions are not assumed, nor necessary, here. 


\section{References}

[1] see e.g. J. Zinn-Justin, "Quantum Field Theory and Critical Phenomena" (1993) Clarendon Press, Oxford, or S. Coleman, "1/N", Erice lectures (1979).

[2] F.J. Wegner and A. Houghton, Phys. Rev. A8 (1973) 401.

[3] S.-K. Ma, Rev. Mod. Phys. 45 (1973) 589.

[4] D.D. Vvedensky, J. Phys. A17 (1984) L251; A20 (1987) L197.

[5] T.S. Chang, D.D. Vvedensky and J.F. Nicoll, Phys. Rep. 217 (1992) 279

[6] N. Tetradis and D.F. Litim, Nucl. Phys. B464 (1996) 492.

[7] M. Reuter, N. Tetradis and C. Wetterich, Nucl. Phys. B401 (1993) 567.

[8] N. Tetradis and C. Wetterich, Nucl. Phys. B422 (1994) 541.

[9] U. Ellwanger and L. Vergara, Nucl. Phys. B398 (1993) 52.

[10] K-I Aoki et al, Prog. Theor. Phys. 95 (1996) 409, hep-ph/9612458.

[11] J. Comellas and A. Travesset, Barcelona preprint UB-ECM-PF 96/21, hep-th/9701028.

[12] T.R. Morris, Int. J. Mod. Phys. A9 (1994) 2411.

[13] M. Turner and T.R. Morris, SHEP 97-06, hep-th/9704202

[14] T.R. Morris, in RG96, SHEP 96-25, hep-th/9610012.

[15] J.F. Nicoll, T.S. Chang and H.E. Stanley, Phys. Rev. Lett. 33 (1974) 540.

[16] T.R. Morris, Phys. Lett. B334 (1994) 355.

[17] T.R. Morris, Nucl. Phys. B458[FS] (1996) 477.

[18] K. Wilson and J. Kogut, Phys. Rep. 12C (1974) 75.

[19] T.R. Morris, Phys. Lett. B329 (1994) 241.

[20] J. Polchinski, Nucl. Phys. B231 (1984) 269.

[21] W.A. Bardeen, M. Moshe and M. Bander, Phys. Rev. Lett. 52 (1984) 1188;

F. David et al, Phys. Rev. Lett. 53 (1984) 2071, Nucl. Phys. B257 (1985) 695.

[22] M. Alford, Phys. Lett. B336 (1994) 237.

[23] J.F. Nicoll and T.S. Chang, Phys. Lett. 62A (1977) 287; C. Wetterich, Phys. Lett. B301 (1993) 90; M. Bonini et al, Nucl. Phys. B409 (1993) 441.

[24] T.R. Morris, in preparation.

[25] T.R. Morris, Southampton preprint 96-36, hep-th/9612117.

[26] R.D. Ball et al, Phys. Lett. B347 (1995) 80.

[27] T.R. Morris, Phys. Rev. Lett. 77 (1996) 1658.

[28] T.R. Morris, Phys. Lett. B357 (1995) 225. 